\title{
Are Dark Energy and Dark Matter Different Aspects of the Same Physical Process?
}

\author{
Ruth E. Kastner ${ }^{1 *}$ and Stuart Kauffman ${ }^{2}$ \\ ${ }^{1}$ Department of Philosophy, University of Maryland, College Park, College Park, MD, United States, ${ }^{2}$ Department of \\ Biochemistry and Biophysics, Institute for Systems Biology, Seattle and Professor Emeritus, University of Pennsylvania, \\ Philade/phia, PA, United States
}

OPEN ACCESS

Edited by:

Ignazio Licata,

ISEM Institute for Scientific

Methodology, Italy

Reviewed by:

Leonardo Chiatti,

Medical Physics Laboratory - Health

Local Authority, Italy

Nicolas Francisco Lori,

Instituto de Pesquisa em Ciências da

Vida e da Saúde (ICVS), Portugal

*Correspondence:

Ruth E. Kastner

rkastner@umd.edu

Specialty section:

This article was submitted to

Interdisciplinary Physics,

a section of the journal

Frontiers in Physics

Received: 15 December 2017

Accepted: 26 June 2018

Published: 13 August 2018

Citation:

Kastner RE and Kauffman S (2018)

Are Dark Energy and Dark Matter

Different Aspects of the Same

Physical Process? Front. Phys. 6:71.

doi: 10.3389/fphy.2018.00071
It is suggested that the apparently disparate cosmological phenomena attributed to so-called "dark matter" and "dark energy" arise from the same fundamental physical process: the emergence, from the quantum level, of spacetime itself. This creation of spacetime results in metric expansion around mass points in addition to the usual curvature due to stress-energy sources of the gravitational field. A recent modification of Einstein's theory of general relativity by Chadwick, Hodgkinson, and McDonald incorporating spacetime expansion around mass points, which accounts well for the observed galactic rotation curves, is adduced in support of the proposal. Recent observational evidence corroborates a prediction of the model that the apparent amount of "dark matter" increases with the age of the universe. In addition, the proposal leads to the same result for the small but non-vanishing cosmological constant, related to "dark energy," as that of the causet model of [1].

Keywords: dark matter, dark energy, cosmological constant, general relativity, spacetime emergence

\section{INTRODUCTION}

Since the 1990s it has become clear that the universe is expanding at an accelerating rate, a phenomenon that was historically attributed to so-called "dark energy"1. The hypothetical dark energy is invisible, and can be thought of as an intrinsic property of spacetime rather than usual matter (stress-energy) that is the source of spacetime curvature. The density of "dark energy" is constant, also in contrast to ordinary matter/energy. A popular method of accounting for the dark energy phenomenon is by attributing it to Einstein's "cosmological constant" $\Lambda$ [3].

An ostensibly separate phenomenon-the flattening of galactic rotation curves with radial distance-is also well known (e.g., [4]). This unexpectedly large value of rotational velocities for the outer observable matter in galaxies is an anomaly for standard Newtonian and Einsteinian gravitational theories, and in order to preserve them, it has been attributed to an invisible hypothetical form of matter dubbed "dark matter." However, rather than postulate "dark matter," some researchers have been exploring modifications of Newtonian gravitational theory. One such effort, "Modified Newtonian Dynamics" or MOND, was introduced by Milgrom [5]. MOND has been successful in fitting the observed rotation curves, but it has the drawback of being an ad hoc alteration to the basic gravitational theory.

The situation has recently progressed significantly: Chadwick et al. [6] have proposed a modification of Einstein's general relativity based on the principle that (idealized) point masses give

\footnotetext{
${ }^{1}$ e.g., Huterer and Turner [2].
} 
rise not only to the usual spacetime curvature, but also to spacetime expansion. For a particular value of the parameter governing the magnitude of the expansion, they find that their theory perfectly fits the galactic rotation data. It should also be noted that their expansion parameter does in principle have a time dependence, although in the approximation studied by them so far, corresponding to the MOND formulation, the time dependence is suppressed.

Currently, there is no known physical mechanism or process underlying the phenomena attributed to dark matter and dark energy (or the finite value of $\Lambda$ if that is an accurate expression of the latter effect). This paper proposes such a physical process: a specific kind of spacetime emergence underlying a form of matter-based spacetime expansion that has not been previously taken into account. Thus, given the quantification of spacetime expansion by the CHM theory, we may be able to physically account for the "dark matter" phenomenon through a previously unsuspected expansion generated by ordinary matter. In addition, "dark energy" may be understood as an artifact of the same emergence process, arising from the discreteness of spacetime and its quantum origins.

We should hasten to note that the current proposal is not itself a theory of quantum gravity, although it may serve as an ontological guide to such a theory. In any case, no particular theory of quantum gravity is required in order for the basic concept to be useful and applicable as a new kind of ontological understanding of the relationship between the quantum level and an emergent spacetime manifold. In what follows, we first review the proposed general framework for spacetime emergence and then show that it naturally leads to the description provided by the CHM theory. Then we discuss another aspect of the emergence process that naturally leads to the non-vanishing, but very small, value of $\Lambda$ that accounts for the "dark energy" phenomenon.

\section{POSSIBLE ORIGIN OF SPACETIME EXPANSION AROUND MASS POINTS}

The present authors have independently proposed that new elements of spacetime emerge from the quantum substratum through a real non-unitary process of measurement, in which quantum potentiae [7] become actualized as new sets of structured spacetime events. One of us, REK, has proposed such a process of actualization and spacetime emergence as a key component of the relativistic extension of the Transactional Interpretation, now called the Relativistic Transactional Interpretation (RTI) (cf. [8], Chapter 8; [9] ${ }^{2}$. The other, SK, has independently been exploring the idea that measurement is a real physical process that converts quantum possibilities (understood as a new metaphysical category, res potentia) to spacetime actualities (identified as Descartes' res extensa) in the context of biophysics ([13], primarily Chapter 7). Both proposals, though having been arrived at and presented in

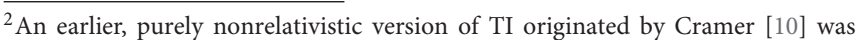
subject to a challenge by Maudlin ([11], 184-5), but that has been completely nullified by the relativistic development resulting in RTI [12].
}

different ways, lead to the same basic idea: spacetime expansion is always associated with "measurement" at the quantum level, understood as a real (but inherently indeterministic) physical process.

In RTI, quantum objects, as described by quantum states, (solutions to the Schrodinger equation or, at the relativistic level, Fock States) are taken as elements of a quantum substratum that is a precursor to spacetime. That is, quantum objects are Heisenbergian potentiae (tokens of res potentia in Kauffman's terminology) that are not spacetime objects. They can be understood as necessary but not sufficient conditions for spacetime events. The transactional process (as detailed, for example, in [8], Chapter 3) is the sufficient condition that results in actualization of a spacetime interval $I$ as defined by an emission event $E$, an absorption event $A$, and the directed temporal and spatial connection between them, which is the transferred quantum (such as a photon).

In this picture, energy and momentum are interpreted physically (not just mathematically) as the generators of temporal and spatial displacement, respectively. Owing to the conjugate nature of the pairs $\{E, t\}$ and $\{P, x\}$, each new interval $I(E, A)$ established by the transfer of $E, P$ (where these are properties of the transferred photon) from $E$ to $A$ is associated with a quantity of action of magnitude $\hbar$. Thus, a new spacetime interval $I(E, A)$ is physically generated as a result of a transaction transferring the conserved physical quantities; one which did not exist before. $I(E, A)$ is distinguishable in the sense that it has in-principle observable properties related to its identification with the process connecting $E$ and $A$ (e.g., energy and directional momentum transferred from $E$ to $A$ ).

An ongoing process of such transactional transfers from emitters and absorbers (i.e., atoms and molecules in the substratum, which can change roles from emitter to absorber and back again by repeatedly becoming excited and decaying) leads naturally to key aspects of the causal set ("causet") model of Sorkin and his collabarators (e.g., [1] and references therein). However, in the RTI picture, each such spacetime event is contingent on the specific physical nature of the transaction that established it. This physically distinguishes and characterizes the spacetime events and their connections, so that they are not just generic "atoms of spacetime" as in the causet model thus far.

More specifics regarding the process of spacetime emergence in the RTI ontology is provided in Kastner [14]. Quantitative results linking specific physical processes to probabilities for "measurement results," including a derivation of the Born Rule for radiative processes (which are actualized transactions), are provided in Kastner and Cramer [15]. It is shown therein and in Kastner [8, 9] that transactions (and thus new structured sets of spacetime events) occur with probabilities associated with decay rates, which are always Poissonian. Interestingly, Bombelli et al. [16] have independently found, with respect to the causet approach, that the growth of the causet in a Poissonian manner preserves Lorentz covariance.

The present proposal differs from that of Sorkin and his collaborators in that the spacetime substratum (i.e., the manifold that is the precursor to the spacetime causet) is comprised of specific quantum entities described by quantum states (i.e., 
field excitations that are created and destroyed). As noted above, these quantum entities stochastically give rise to new elements of the causet in a Poissonian process [14]. In this picture, there are many possible (candidate) events for addition to the spacetime causet, but there is just one actual growing causet, and that is the emergent spacetime. The structure of that growing spacetime is contingent on the specific quantum entities (and their interactions) in the substratum; thus, it is those which will dictate the transition probabilities from a causet with $\mathrm{N}$ elements to a larger one with $\mathrm{N}+1$ elements, rather than a transition probabilities applying to an arbitrary Markov process as in the classical sequential growth model (intended as a first step toward a quantum version of causet growth) studied in Rideout and Sorkin [1]. Nevertheless, the fact that the uncertainty $\Delta \mathrm{N}$ in the number of elements $\mathrm{N}$ is Poissonian leads to the same prediction for the cosmological constant as found by [1], and therefore a physical basis for "dark energy"; we turn to that in section The cosmological constant and "dark energy." First, however, we should note that in the RTI picture (as opposed to the approach of [1]), a theory of "quantum gravity" consists of quantifying the correspondence between the elements of the quantum substratum and the emergent spacetime causet structure, the latter being the gravitational metric. A promising way forward in this regard is through the poset work of Knuth et al. (e.g., [17]).

How can we understand the new spacetime interval created in an actualized transaction as a form of spacetime expansion around a mass point, in order to find correspondence with the CHM theory accounting for "dark matter"? At the quantum level, a "mass point" would be something like an isolated atom; say a hydrogen atom $A$. According to the current proposal, the atom is part of the quantum substratum-not a spacetime objectunless it is "measured" i.e., engages in a transaction in terms of RTI. In order for $A$ to count as a persistent mass point that could serve a source of stress-energy, it would have to be subject to ongoing measurement-engaging in transactions that enable it to approximate a spacetime trajectory (see, e.g., [8], section 4.4$)^{3}$. These ongoing transactions (arising from other emitters and absorbers in the universe including Earth-based astronomical equipment) serve to repeatedly actualize $A$, and with every actualization, a new spacetime interval is created that did not exist before. This results in an observable expansion of the metric in the locus of $A$, in addition to any curvature already accounted for in standard general relativity. Note that the expansion is not confined to the spatial domain, but includes the temporal domain as well (this is implicit in the CHM theory).

We therefore obtain a specific (although at this stage, qualitative) prediction: the expansion effect attributed to a specific quantity of "dark matter" should increase monotonically with increasing proper time of the universe $\tau$ In fact, such an effect has just recently been observed: very distant (i.e., large

\footnotetext{
${ }^{3}$ This process of a quantum system approximating a classical trajectory through measurement is well-known (not solely an aspect of RTI) and is related to the well-known "inverse Zeno effect" (see, e.g., [18]).
}

redshift, and therefore very young, recently born) galaxies have rotation curves much closer to the Newtonian gravitational prediction than do older galaxies [19]. (Of course, Genzel et al. [19] interpret the data based on the usual assumption that "dark matter" really exists; they therefore tentatively conclude that the difference has to do with less "dark matter" in the past in relation to the amount of normal baryonic matter). We take this is a tentative corroboration of the model, but of course more observations are called for. In particular, it is now possible to study dark matter as a function of the age of a galaxy, and in addition, it may be possible to ascertain whether dark matter is spatially isotropic, or shows any variation with the density of observable matter.

\section{THE COSMOLOGICAL CONSTANT AND "DARK ENERGY"}

We now return to the issue of "dark energy." As noted above, the result of the transactional spacetime emergence process is to yield a causal set of the sort contemplated by [1], although the elements of the set have more structure in this picture; they are networked transactions $I\left(E_{i}, A_{j}\right)$ (where the indices are a shorthand representing birth order, chain membership, conserved physical quantities transferred, etc. ${ }^{4}$ ). In this regard, they more closely resemble the "influence network" of Knuth et al. (e.g., [20]). Nevertheless, the fact that elements of causet are added in Poissonian fashion means that the current model yields the same non-vanishing, but very tiny, value for $\Lambda$.

Specifically, in natural units $(h=G=1) \Lambda$ has units of inverse length squared, and observations indicate that

$$
\Lambda \lesssim 1 / \mathrm{V}^{1 / 2}
$$

Based on empirical data, $\Lambda$ must be very close to zero; but to a first order approximation, one might find a very small but non-negligible value ${ }^{5}$. Sorkin [22] provides such a first-order approximation, as follows. One notes (based on unimodular gravity $^{6}$ ) that $\Lambda$ and $\mathrm{V}$ are essentially conjugate; i.e.,

$$
\Delta \Lambda \Delta V \sim 1
$$

(in natural units), analogously to the quantum mechanical uncertainty relations. Sorkin notes that this conjugate relationship between $\Lambda$ and $\mathrm{V}$ is evident from the action integral,

$$
S=-\Lambda \int(-g)^{1 / 2} d^{4} x=-\Lambda V
$$

Thus, if $\Lambda$ has a non-vanishing value, it may be due to its uncertainty

$$
\Delta \Lambda \sim 1 / \Delta \mathrm{V}
$$

\footnotetext{
${ }^{4}$ A "chain" is a subset of a causet possessing a total order of its elements, providing a timelike relationship among them.

${ }^{5}$ For a discussion of the puzzle of small $\Lambda$, see $\mathrm{Ng}$ and van Dam [21].

${ }^{6}$ I.e., the condition that the metric tensor $g$ has unit determinant.
} 
based on any uncertainty in V. In the causet model, $\mathrm{V}$ is proportional to the number of elements $\mathrm{N}$, since the latter specifies how many "atoms of spacetime" exist; or, in the RTI picture, how many $I\left(E_{i}, A_{j}\right)$ have been actualized. Now, given that elements are added to the (discrete) spacetime manifold in a Poissonian process, the number $\mathrm{N}$ of elements has an intrinsic uncertainty of $\mathrm{N}^{1 / 2}$ for any given value of the proper time $\tau$. Since $\mathrm{V}$ is a function of $\tau, \mathrm{V}$ inherits this uncertainty: $\Delta \mathrm{V} \sim$ $\mathrm{V}^{1 / 2}$. If the uncertainty is the only (significant) contribution to the value of $\Lambda$, then we get precisely (1).

\section{CONCLUSION}

We have proposed a specific mechanism of spacetime emergence from the quantum level that leads to the spacetime expansion quantitatively described in the theory of Chadwick et al. [6], which correctly predicts observed galaxy rotation data attributed to "dark matter." In addition, we have shown that the same mechanism yields a discrete spacetime characterized by Poissonian uncertainties, similar to that proposed by [1], which results in the necessary value of $\Lambda$ to account for the "dark energy" phenomenon, according to current observational data.

\section{REFERENCES}

1. Rideout DP, Sorkin RD. A classical sequential growth model for causal sets. Phys Rev. (2000) D61:024002. doi: 10.1103/PhysRevD.61. 024002

2. Huterer D, Turner MS. Prospects for probing the dark energy via supernova distance measurements. Phys Rev D (1999) 60: 1-5.

3. Einstein A. Kosmologische Betrachtungen zur allgemeinen Relativitaetstheorie. In: Sitzungsberichte der Königlich Preussischen Akademie der Wissenschaften Part 1. Berlin. (1917). p. 142-52.

4. Rubin V, Thonnard N, Ford WK Jr. Rotational properties of $21 \mathrm{Sc}$ galaxies with a large range of luminosities and radii from NGC 4605 ( $R=4 \mathrm{kpc})$ to UGC 2885 (R=122kpc). Astrophys J. (1980) 238:471-87.

5. Milgrom M. A modification of the Newtonian dynamics as a possible alternative to the hidden mass hypothesis. Astrophys J. (1983) 270:365.

6. Chadwick EA, Hodgkinson TF, McDonald GS. A gravitational development supporting MOND. Phys Rev. (2013) D88:024036. doi: 10.1103/PhysRevD.88.024036

7. Heisenberg W. Physics and Philosophy. New York, NY: Harper-Row (1958).

8. Kastner RE. The Transactional Interpretation of Quantum Mechanics: The Reality of Possibility. Cambridge: Cambridge University Press (2012).

9. Kastner RE. The possibilist transactional interpretation and relativity. Found Phys. (2012) 42:1094-113. doi: 10.1007/s10701-012-9658-4

10. Cramer J. The transactional interpretation of quantum mechanics. Rev Mod Phys. (1986) 58:647-88.

11. Maudlin T. Quantum Nonlocality and Relativity. 3rd ed. Oxford: Blackwell (2011).

12. Kastner RE. The relativistic transactional interpretation: immune to the maudlin challenge. In: Aerts D, Dalla Chiara ML, de Ronde C, Krause D, editors. Probing the Meaning and Structure of Quantum Mechanics. Singapore: World Scientific (2017).

13. Kauffman S. Humanity in a Creative Universe. Oxford: Oxford University Press (2016).

14. Kastner RE. The emergence of spacetime: transactions and causal sets. In: Licata I. editor. Beyond Peaceful Coexistence. Singapore: World Scientific (2016). p. arXiv:1411.2072.
In this model, we may understand "dark energy" as a property arising from the ever-present basic quantum uncertainty in the spacetime volume $\mathrm{V}$.

This possible relation of dark energy and matter is intriguing, as it would unify apparently disparate and yet equally unexpected cosmological phenomena. If an expansion of spacetime around mass points can account for the excess rotation of the outskirts of galaxies (i.e., "dark matter"), and if this expansion is related to dark energy as outlined herein, we gain explanatory parsimony as well as evidence for a fascinating connection of spacetime with the quantum level. The latter could aid efforts to find a theory of quantum gravity.

\section{AUTHOR'S NOTE}

A preprint form of this paper is available online at https://arxiv. org/abs/1708.02907. The authors hold the copyright to this paper.

\section{AUTHOR CONTRIBUTIONS}

SK shared formation and writing of MS with RK in entirety.

15. Kastner RE, Cramer JG. Quantifying Absorption in the Transactional Interpretation (2018). Available online at: https://arxiv.org/abs/1711.04501

16. Bombelli L, Henson J, Sorkin RD. Discreteness without symmetry breaking: a theorem. Mod Phys Lett. (2006) A24:2579-87. doi: 10.1142/S0217732309031958

17. Walsh JL, Knuth KH. An information physics derivation of equations of geodesic form from the influence network. In: MaxEnt 2015 Conference, Bayesian Inference and Maximum Entropy Methods in Science and Engineering, Potsdam NY (2015). Available online at: https://arxiv.org/abs/ 1604.08112

18. Panov A. Inverse Quantum Zeno Effect in Quantum Oscillations. (2001). Available online at: http://cds.cern.ch/record/515461/files/0108130.pdf

19. Genzel R, Schreiber NM, Übler H, Lang P, Naab T, Bender R, et al. Strongly baryon-dominated disk galaxies at the peak of galaxy formation ten billion years ago. Nature (2017) 543:397-401. doi: 10.1038/nature21685

20. Knuth K, Bahreyni N. A potential foundation for emergent space-time. J Math Phys. (2014) 55:112501. doi: 10.1063/1.4899081

21. Ng J, van Dam H. A small but nonzero cosmological constant. Int J Mod Phys (2001) D10:49-56. doi: 10.1142/S0218271801000627

22. Sorkin RD. Is the cosmological "constant" a nonlocal quantum residue of discreteness of the causal set type? AIP Conf. Proc. (2007) 957:142-53. doi: $10.1063 / 1.2823750$

Conflict of Interest Statement: The authors declare that the research was conducted in the absence of any commercial or financial relationships that could be construed as a potential conflict of interest.

The handling editor and reviewer LC declared their involvement as co-editors in the Research Topic, and confirm the absence of any other collaboration.

Copyright (c) 2018 Kastner and Kauffman. This is an open-access article distributed under the terms of the Creative Commons Attribution License (CC BY). The use, distribution or reproduction in other forums is permitted, provided the original author(s) and the copyright owner(s) are credited and that the original publication in this journal is cited, in accordance with accepted academic practice. No use, distribution or reproduction is permitted which does not comply with these terms. 